\title{
Rapid immunochromatography-based detection of carbapenemase producers
}

\author{
Nicolas Kieffer ${ }^{1,2,3} \cdot$ Laurent Poirel $^{1,2,3} \cdot$ Patrice Nordmann ${ }^{1,2,3,4}$
}

Sir,

The increasing spread of carbapenemase-producing Gramnegative bacteria represents one of the major public health problems. The main carbapenemases identified in clinical isolates belong to different structural types of $\beta$-lactamases belonging to three different classes: the Klebsiella pneumoniae Carbapenemase (KPC) enzymes belong to the class A, the New Delhi Metallo- $\beta$-lactamase (NDM), the Verona integron-encoded metallo- $\beta$-lactamase (VIM) and the imipenemase (IMP) enzymes belong to the class $\mathrm{B}$, and the OXA-48-types belong to the class D. One of the most efficient ways to limit the dissemination of carbapenemaseproducing isolates is their early detection in patients being infected or colonized, to rapidly implement adequate control measures.

Recent and rapid carbapenemase detection tests are currently available and are based on the biochemical detection of the carbapenem hydrolysis [1, 2]. Recently, an immunochromatographic detection test, the NG-test Carba 5 (NG Biotech, Guipry, France), has been developed. This lateral flow technique allows the detection of the five main carbapenemase types, i.e., KPC, IMP, VIM, NDM, and OXA-48types (OXA-48, OXA-162, OXA-181, OXA-204, OXA-232, and OXA-244). This kit detects most of the variants of these

Patrice Nordmann

patrice.nordmann@unifr.ch

1 Medical and Molecular Microbiology Unit, Department of Medicine, Faculty of Science and Medicine, University of Fribourg, Chemin du Musée 18, 1700 Fribourg, Switzerland

2 INSERM European Unit (IAME, France), University of Fribourg, Fribourg, Switzerland

3 Swiss National Reference Center for Emerging Antibiotic Resistance (NARA), University of Fribourg, Fribourg, Switzerland

4 Institute for Microbiology, University of Lausanne and University Hospital Centre, Lausanne, Switzerland families except the class D carbapenemases OXA-23, OXA40, and OXA-58 that are identified in Acinetobacter spp. isolates. As part of the mission of our national reference center in Switzerland, we have evaluated this test with a collection of carbapenemase-producing Enterobacteriaceae and non-Enterobacteriaceae isolates, and additionally with double-carbapenemase producers that may be also identified.

We tested a collection of 73 carbapenem-resistant Gramnegative bacilli. This collection included 45/73 Enterobacteriaceae (Citrobacter freundii, Enterobacter aerogenes, Enterobacter cloacae, Escherichia coli, Klebsiella pneumoniae, Klebsiella oxytoca, Proteus mirabilis, and Serratia marcescens), 11/73 Acinetobacter baumannii and 17/73 Pseudomonas aeruginosa. The isolates of the collection were resistant to carbapenems due to different mechanisms: (1) production of a carbapenemase (OXA-48-types $n=15$, KPC $n=9$, NDM $n=13$, VIM $n=14$, IMP $n=9$, OXA-23 $n=23$, OXA-40 $n=2$ and OXA-58 $n=2$ ), (2) overproduction of a cephalosporinase associated with permeability defects $(n=4)$ or $(3)$ impermeability alone $(n=3)$ (Table 1$)$. Noteworthy, four isolates co-produced two carbapenemases (NDM-1 and OXA-48-types) (Table 1). All carbapenemase genes were identified by PCR experiments followed by sequencing.

All isolates were grown on URISelect ${ }^{\mathrm{TM}} 4$ agar plates (Bio-Rad, Cressier, Switzerland) for $16 \mathrm{~h}$ at $37{ }^{\circ} \mathrm{C}$. The NG-test Carba 5 kit was used according to the manufacturer's recommendations. Briefly, one colony was mixed with five drops of lysis buffer included in the test and $100 \mu \mathrm{l}$ of the lysis suspension was added to the immunological lateral flow cassette. The results were obtained after $15 \mathrm{~min}$ of incubation at room temperature and are reported in Table 1. The NG-test Carba 5 kit allowed the identification of all KPC, OXA-48-types, NDM, VIM and IMP-producers. Noteworthy, this test was able to detect both carbapenemases in the isolates co-producing both NDM-1 and OXA-48-types. As expected, this test cannot detect the most important carbapenemases in A. baumannii 
Table 1 Results of the NG-test Carba 5 kit

\begin{tabular}{|c|c|c|c|}
\hline Species & Resistance determinant & $\begin{array}{l}\text { Number of } \\
\text { isolates }\end{array}$ & Result \\
\hline \multicolumn{4}{|l|}{ OXA-48-type } \\
\hline \multirow[t]{3}{*}{ K. pneumoniae } & OXA-181 & 2 & + \\
\hline & OXA-162 & 1 & + \\
\hline & OXA-48 & 2 & + \\
\hline \multirow[t]{2}{*}{ E. coli } & OXA-244 & 1 & + \\
\hline & OXA-204 & 1 & + \\
\hline E. cloacae & OXA-181 & 1 & + \\
\hline E. aerogenes & OXA-48 & 1 & + \\
\hline S. marcescens & OXA-48 & 1 & + \\
\hline C. freundii & OXA-162 & 1 & + \\
\hline P. aeruginosa & OXA-181 & 1 & + \\
\hline \multicolumn{4}{|l|}{$\mathrm{KPC}$} \\
\hline \multirow[t]{3}{*}{ K. pneumoniae } & KPC-11 & 2 & + \\
\hline & KPC-3 & 1 & + \\
\hline & KPC-2 & 1 & + \\
\hline \multirow[t]{2}{*}{ E. coli } & KPC-3 & 1 & + \\
\hline & KPC-2 & 1 & + \\
\hline E. cloacae & KPC-2 & 1 & + \\
\hline P. aeruginosa & KPC-2 & 2 & + \\
\hline \multicolumn{4}{|l|}{ NDM } \\
\hline K. pneumoniae & NDM-7 & 1 & + \\
\hline K. oxytoca & NDM-1 & 1 & + \\
\hline \multirow[t]{3}{*}{ E. coli } & NDM-7 & 1 & + \\
\hline & NDM-6 & 1 & + \\
\hline & NDM-5 & 2 & + \\
\hline C. freundii & NDM-5 & 1 & + \\
\hline \multirow[t]{2}{*}{ A. baumannii } & NDM-2 & 1 & + \\
\hline & NDM-1 & 1 & + \\
\hline P. aeruginosa & NDM-1 & 1 & + \\
\hline \multicolumn{4}{|l|}{ VIM } \\
\hline \multirow[t]{2}{*}{ K. pneumoniae } & VIM-4 & 1 & + \\
\hline & VIM-1 & 2 & + \\
\hline \multirow[t]{3}{*}{ E. coli } & VIM-19 & 1 & + \\
\hline & VIM-2 & 1 & + \\
\hline & VIM-1 & 1 & + \\
\hline E. cloacae & VIM-1 & 2 & + \\
\hline P. mirabilis & VIM-1 & 1 & + \\
\hline \multirow[t]{3}{*}{ P. aeruginosa } & VIM-4 & 1 & + \\
\hline & VIM-2 & 3 & + \\
\hline & VIM-1 & 1 & + \\
\hline \multicolumn{4}{|l|}{ IMP } \\
\hline K. pneumoniae & IMP-8 & 1 & + \\
\hline \multirow[t]{2}{*}{ S. marcescens } & IMP-11 & 1 & + \\
\hline & IMP-1 & 1 & + \\
\hline A. baumannii & IMP-5 & 1 & + \\
\hline
\end{tabular}

Table 1 (continued)

\begin{tabular}{|c|c|c|c|}
\hline Species & Resistance determinant & $\begin{array}{l}\text { Number of } \\
\text { isolates }\end{array}$ & Result \\
\hline \multirow[t]{5}{*}{ P. aeruginosa } & IMP-29 & 1 & + \\
\hline & IMP-19 & 1 & + \\
\hline & IMP-15 & 1 & + \\
\hline & IMP-10 & 1 & + \\
\hline & IMP-1 & 1 & + \\
\hline \multicolumn{4}{|c|}{ Double carbapenamase } \\
\hline \multirow[t]{3}{*}{ K. pneumoniae } & NDM-1 + OXA-48 & 1 & + \\
\hline & NDM-1 + OXA-181 & 1 & + \\
\hline & NDM-1 + OXA-232 & 2 & + \\
\hline \multicolumn{4}{|l|}{ Negative control } \\
\hline E. cloacae & AmpC & 2 & - \\
\hline E. aerogenes & $\mathrm{AmpC}$ & 1 & - \\
\hline S. marcescens & $\mathrm{AmpC}$ & 1 & - \\
\hline \multirow[t]{4}{*}{ A. baumannii } & AmpC & 1 & - \\
\hline & OXA-23 & 3 & - \\
\hline & OXA-40 & 2 & - \\
\hline & OXA-58 & 2 & - \\
\hline P. aeruginosa & Impermeability & 3 & - \\
\hline
\end{tabular}

that are OXA-23, OXA-40, and OXA-58. No false positive was observed among the collection tested. Altogether, the values of sensitivity and specificity of this test were $100 \%$. These results are in full agreement with a previous evaluation that has been published since our work was in progress [3].

The NG-test Carba 5 detection kit is a recent immunochromatographic test based on the recognition of conserved epitopes belonging to five major families of carbapenemases identified in clinical Enterobacteriaceae, A. baumannii and $P$. aeruginosa isolates. Besides its simplicity to use, this test gives results of the presence of carbapenemases within $15 \mathrm{~min}$, even for the OXA-48-type producers that biochemical tests struggle to detect. This test can be used for a diversity of clinical species including Enterobacteriaceae, A. baumannii, and $P$. aeruginosa. Another immunochromatographic test has been developed recently, namely the O.K.N.V k-set test (Coris BioConcept, Belgium) [4]. This test is based on the same technology of the NG-test Carba 5 but lack the detection of the IMP carbapenemases. Furthermore, the O.K.N.V k-set test failed to detect some chromosomally encoded carbapenemases such as NDM in $P$. mirabilis [5].

An additional limitation point of these immunochromatographic tests is their ability to detect only known enzymes compared to biochemical tests based on a color change such as the Rapidec CarbaNP ${ }^{\circledR}$ or the $\beta$-carba test ${ }^{\circledR}[1,2]$ that can detect any carbapenemase activity, including potential new enzymes. Nevertheless, we believed that this test may be 
useful as a second-line test after usage of the broad-spectrum carbapenemase screening rapid test based on biochemistry.

In summary, the NG-test carba 5 represents a reliable tool to detect the production of the five most common carbapenemase families identified in Gram-negative bacteria and showed a high sensitivity even towards different variants of these enzymes.

Acknowledgements We are grateful to Anthony Demord and Laura Vazquez-Rojo for the technical support.

Funding This work was funded by the Swiss National Reference Center for Emerging Antibiotic Resistance (NARA).

\section{Compliance with ethical standards}

Conflict of interest The authors declare that they have no conflict of interest.

\section{References}

1. Nordmann P, Poirel L, Dortet L. Rapid detection of carbapenemase-producing Enterobacteriaceae. Emerg Infect Dis. 2012;18:1503-7.

2. Mancini S, Kieffer N, Poirel L, Nordmann P. Evaluation of the RAPIDEC ${ }^{\circledR}$ CARBA NP and $\beta$-CARBA ${ }^{\circledR}$ tests for rapid detection of Carbapenemase-producing Enterobacteriaceae. Diagn Microbiol Infect Dis. 2017;88:293-7.

3. Hopkins KL, Meunier D, Naas T, Volland H, Woodford N. Evaluation of the NG-Test CARBA 5 multiplex immunochromatographic assay for the detection of KPC, OXA-48-like, NDM, VIM and IMP carbapenemases. J Antimicrob Chemother. 2018;73:3523-6.

4. Kolenda C, Benoit R, Carricajo A, Bonnet R, Dauwalder O, Laurent F. Evaluation of the new multiplex immunochromatographic O.K.N.V. k-SeT assay for rapid detection of OXA-48like, KPC, NDM, and VIM carbapenemases. J Clin Microbiol. 2018;56:e1247-18. https://doi.org/10.1128/JCM.01247-18.

5. Saleh A, Göttig S, Hamprecht AG. Multiplex immunochromatographic detection of OXA-48, KPC, and NDM carbapenemases: impact of inoculum, antibiotics, and agar. J Clin Microbiol. 2018;56:e00050-18. https://doi.org/10.1128/JCM.00050-18. 